At 4 months after the initiation of treatment, the mortality rates for the VAD and Cy-Dex groups were $5.8 \%$ and $1.9 \%$, respectively $(P=0.08)$. Compared with the VAD group, significantly fewer toxicity-related events were reported in the Cy-Dex group $(P<0.0001)$. Partial or better response rates after ASCT were comparable in the two groups $(80 \%$ in the VAD arm, and $81 \%$ in the Cy-Dex arm), while $36 \%$ of the VAD arm and $32 \%$ of the Cy-Dex arm showed complete responses. In both groups, the median event-free survival at a median follow-up of 22 months was 29 months and the overall survival at 3 years was $75 \%$. The results of this study show that Cy-Dex is a safe and efficient initial therapy before ASCT for patients with multiple myeloma.

Original article Mellqvist UH et al. (2007)

Cyclophosphamide plus dexamethasone is an efficient initial treatment before high-dose melphalan and autologous stem cell transplantation in patients with newly diagnosed multiple myeloma: results of a randomized comparison with vincristine, doxorubicin, and dexamethasone. Cancer 112: 129-135

\section{Adjuvant radiotherapy benefits patients with early-stage pancreatic cancer}

The survival prospects for patients with pancreatic cancer remain poor, despite improvements in surgical techniques and the application of adjuvant chemotherapy and/or radiotherapy. Trials show that patients given adjuvant chemotherapy gain a survival advantage, but recent studies have reported that radiation therapy, although commonly implemented, might have little impact on survival.

Artinyan et al. analyzed the effect of adjuvant external-beam radiation therapy (EBRT) in patients with pancreatic cancer who had local, lymph-node-negative (NO) tumors. Patients with systemic disease were excluded. By use of the Surveillance, Epidemiology, and End Results registry, the authors identified 1,930 patients with NO disease who had undergone surgery, and used statistical methods to compare overall survival between patients who underwent EBRT and those who did not.

Patients survived for a median of 17 months; those who had received EBRT had significantly better survival (20 months) than those who did not receive radiation (15 months). Multivariate analysis showed that adjuvant EBRT was an independent indicator of survival, as were age at diagnosis, tumor grade, tumor classification and tumor location. When the authors excluded patients who survived less than 3 months after treatment, no survival difference was noted between the two groups on univariate analysis. Multivariate analysis in this patient subgroup, however, revealed EBRT as an independent predictor of better overall survival.

The authors conclude that adjuvant EBRT can improve survival in patients with $\mathrm{NO}$ tumors that are treatable by surgical resection. Although EBRT confers little benefit on patients with late-stage pancreatic cancer, the authors suggest that it might benefit patients with early-stage NO disease.

Original article Artinyan A et al. (2007) Improved survival with adjuvant external-beam radiation therapy in lymph node-negative pancreatic cancer. Cancer 112: 34-42

\section{Interferon treatment reduces incidence of malignant lymphoma in patients infected with HCV}

Chronic infection with hepatitis $\mathrm{C}$ virus (HCV) can lead to cirrhosis and is a risk factor for hepatocellular carcinoma. In addition, antiHCV seropositivity is a known risk factor for malignant lymphoma. However, although many reports suggest a link between HCV infection and malignant lymphoma, few have investigated whether interferon therapy reduces the risk of this type of cancer. Kawamura et al. investigated this issue by performing a retrospective study of patients with HCV infection.

The incidence rates of malignant melanoma were assessed in $501 \mathrm{HCV}$-infected patients who had never received interferon therapy, and in 2,708 HCV-infected patients who had been actively treated with interferon. Cumulative rates of malignant lymphoma development in patients who had never received interferon were $0.6 \%$ at the end of 5 years, $2.3 \%$ at the end of 10 years and $2.6 \%$ at the end of 15 years. Rates among patients who had received interferon treatment but whose infection persisted despite therapy were lower $-0.16 \%$ at 5 years, $0.61 \%$ at 10 years and $1.81 \%$ at 15 years. The most impressive finding was that patients whose HCV load was effectively cleared by interferon treatment did not develop malignant lymphoma, even after 15 years. 